\title{
Virtual anatomy and pathoanatomy - Subluxation fracture of the cervical spine
}

\author{
Valentin Derler' ${ }^{*}$, Franz A Fellner ${ }^{1,2}$ \\ ${ }^{1}$ Central Radiology Institute, Kepler University Hospital, Medical Faculty of the Johannes Kepler University, Linz, Austria \\ ${ }^{2}$ Medical Faculty of the Friedrich-Alexander-University of Erlangen-Nürnberg, Erlangen, Germany
}

\section{Case report}

We report a 49-year-old patient who was admitted to the hospital after a fall from a horse. The patient had radicular symptoms in dermatome $\mathrm{C} 6$ on the right with pain and tingling paraesthesia without motor deficit. The shockroom CT revealed mainly a horizontal fracture in segment $\mathrm{C6} / 7$ with course through the spinosus process $\mathrm{C} 6$, cover plate impression fracture $\mathrm{C} 7$ and traumatic anterolisthesis $\mathrm{C6} / 7$ (Figure 1).

Subsequent MR imaging of the cervical spine revealed a discoligamentous injury in the C6/7 segment without proof of myelopathy (Figure 2).

To illustrate the virtual anatomy and pathoanatomy of this lesion, a three-dimensional reconstruction using Cinematic Rendering was created from the standard CT data set in addition to the standard reconstructions (Figure 3).

The unstable fracture was immediately surgically treated by an anterior discectomy with interbody fusion and anterior plate and screw fixation. Figure 4 shows the postoperative conventional $x$-ray of the cervical spine. After an uncomplicated course, the patient could be released from inpatient treatment without a neurological deficit.

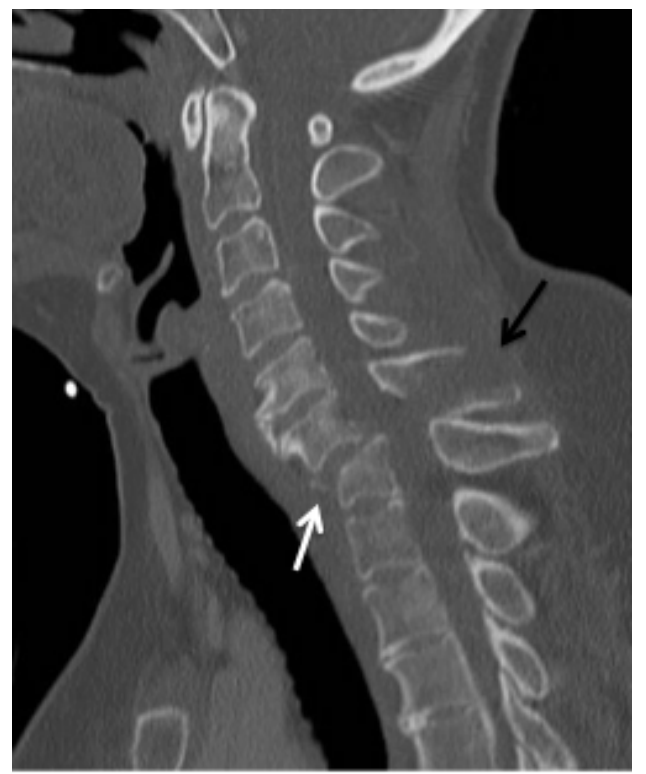

Figure 1. CT of the cervical spine with cover plate impression fracture $\mathrm{C} 7$, traumatic anterolisthesis C6 (white arrow), and horizontal fracture of the spinosus process C6 (black arrow)

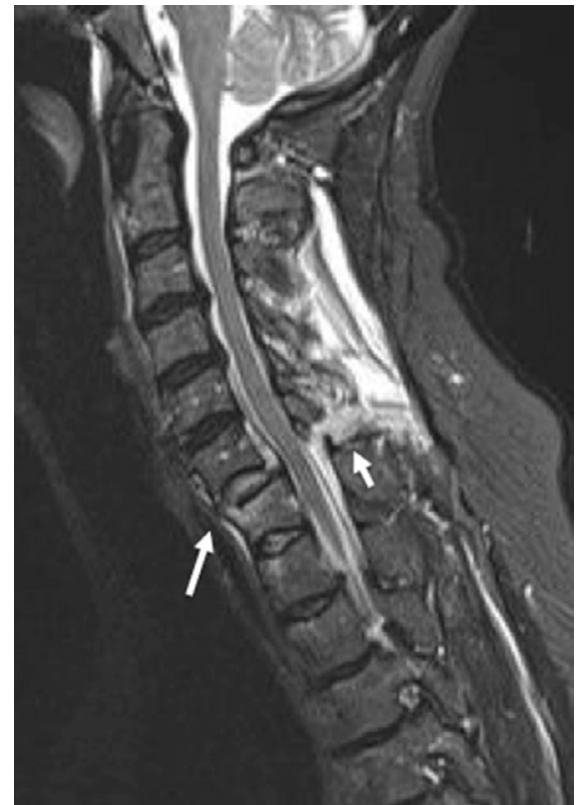

Figure 2. MR imaging of the cervical spine (STIR sequence) with discoligamentous injury in segment C6/7 (arrows)

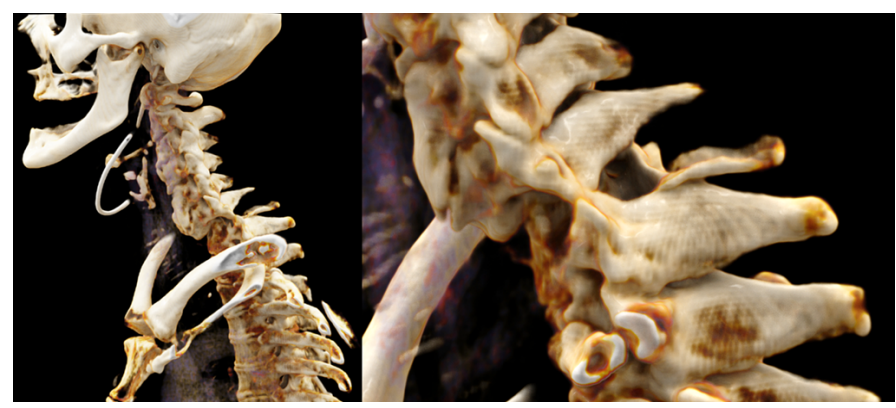

Figure 3. Cinematic Rendering of the subluxation fracture $\mathrm{C} 6 / 7$ based on the CT examination

*Correspondence to: Valentin Derler, Central Radiology Institute, Kepler University Hospital, Medical Faculty of the Johannes Kepler University, Linz, Austria, E-mail: valentin.derler@kepleruniklinikum.at

Key words: virtual anatomy, virtual pathoanatomy, cinematic rendering, subluxation fracture, cervical spine

Received: March 12, 2020; Accepted: April 13, 2020; Published: April 16, 2020 


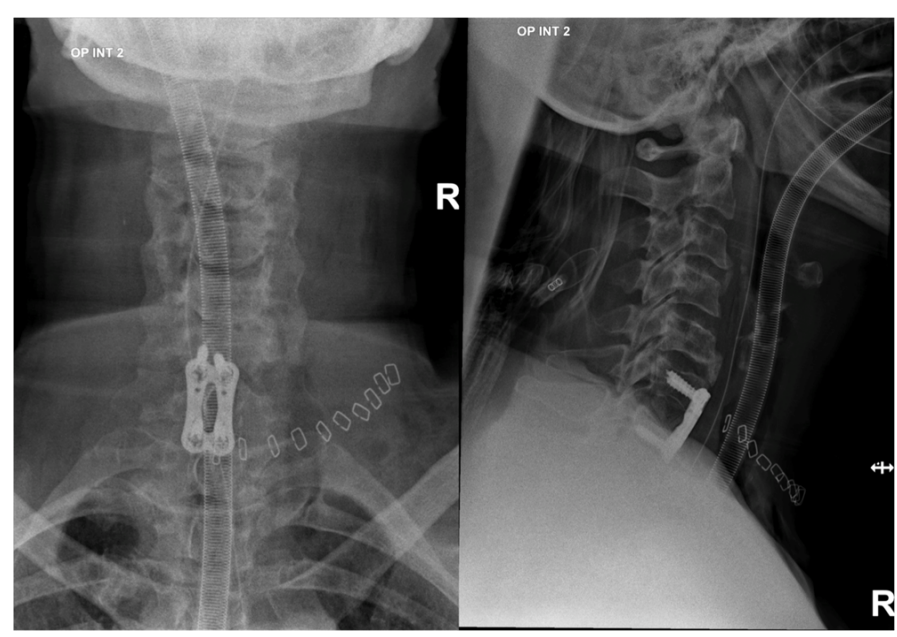

Figure 4. Plain x-ray of the cervical spine after osteosynthesis

\section{Discussion}

Cinematic Rendering (CR) is a recent technique of digital post-processing, which was inspired by animated films from the entertainment industry. In contrast to established methods, such as Volume Rendering, Cinematic Rendering enables an extremely photorealistic representation of anatomical and pathoanatomical structures [1]. The technology is based on an algorithms that take into account the complex propagation of light waves and the interaction of photons with the human anatomy thus simulating realistic light and shadow effects. Cinematic Rendering is particularly suitable for displaying structures with high density or high contrast, such as bone $[1,2]$.

A previous publication emphasized the photo-realistic illustration of fractures [3]. A recent study found a significantly more detailed representation of intra-articular multi-fragment fractures compared to Volume Rendering [4]. This case report illustrates these benefits of Cinematic Rendering for visualization of a complex cervical spine fracture in addition to the conventional methods. Cinematic Rendering has also been used in medical fields other than traumatology. For example, several publications have addressed abdominal lesions and tumors [5-7]. So it is assumed that Cinematic Rendering has the potential to improve the detection and assessment of hepatic lesions [5]. It is stated that Cinematic Rendering could simplify preoperative planning and intraoperative decision making for surgeons and could be used for demonstration in interdisciplinary conferences $[2,4,8]$.

Further studies are necessary to assess the use and benefits of Cinematic Rendering in a clinical context. For teaching medical students we already use Cinematic Rendering in addition to dissection courses and see that it contributes to a better anatomical understanding in anatomy lessons. In this sense, non-medical health professions also benefit from the use of Cinematic Rendering [9-11].

\section{Disclosure}

No conflicts of interest. No grants or financial supports.

\section{References}

1. Fellner FA(2016) Introducing cinematic rendering: A novel technique for postprocessing medical imaging data. J Biomedical Science and Engineering 9: 170-175.

2. Eid M, De Cecco CN, Nance JW, Caruso D, Albrecht, MH, et al. (2017) Cinematic Rendering in CT: A Novel, Lifelike 3D Visualization Technique. American Journal of Roentgenology 209(2): 370-379.

3. Rowe SP, Fritz J, Fishman EK (2018) CT evaluation of musculoskeletal trauma: initial experience with cinematic rendering. Emerg Radiol 25(1): 93-101.

4. Wollschlaeger LM, Boos J, Jungbluth P (2020) Is CT-based cinematic rendering superior to volume rendering technique in the preoperative evaluation of multifragmentary intraarticular lower extremity fractures? Eur J Radiol 126: 108911.

5. Chu LC, Rowe SP, Fishman EK (2019) Cinematic rendering of focal liver masses Diagn Interv Imaging 100(9): 467-476.

6. Rowe SP, Chu LC, Meyer AR, Gorin MA, Fishman EK (2019) The application of cinematic rendering to CT evaluation of upper tract urothelial tumors: principles and practice. Abdom Radiol (NY) 44(12): 3886-3892.

7. Rowe SP, Chu LC, Fishman EK (2019) 3D CT cinematic rendering of the spleen: Potential role in problem solving. Diagn Interv Imaging 100(9): 477-483.

8. Elshafei M, Binder J, Baecker J (2019) Comparison of Cinematic Rendering and Computed Tomography for Speed and Comprehension of Surgical Anatomy. JAMA Surg 154(8): 738-744.

9. Binder J, Krautz C, Engel K, Grützmann R, Fellner FA, et al. (2019) Leveraging medical imaging for medical education - a cinematic rendering-featured lecture. Ann Anat 222: 159-165.

10. Fellner FA, Berger F, Fellner C, Kremer C, Hortner H, et al. (2016) Volume rendering of medical imaging data for the general public. J Health Med Informat 7: 213.

11. Fellner FA, Engel K, Kremer C (2017) Virtual Anatomy: The dissecting theatre of the future - implementation of cinematic rendering in a large $8 \mathrm{~K}$ high-resolution projectionenvironment. J Biomedical Science and Engineering 10: 367-375.

Copyright: (C2020 Derler V. This is an open-access article distributed under the terms of the Creative Commons Attribution License, which permits unrestricted use, distribution, and reproduction in any medium, provided the original author and source are credited. 